\title{
Altered neurochemical coupling in the occipital cortex in migraine with visual aura
}

Running head: Neurochemistry of migraine

Holly Bridge ${ }^{* 1,2}$, DPhil, Charlotte J Stagg 1,2,3, DPhil, Jamie Near ${ }^{4}$ PhD, Aimee Zisner² MSc., Zameel Cader DPhil MRCP1

${ }^{1}$ Nuffield Department of Clinical Neuroscience, University of Oxford

2 Oxford Centre for functional MRI of the Brain (FMRIB), University of Oxford

3 Oxford Centre for Human Brain Activity, University of Oxford

${ }^{4}$ Department of Psychiatry, McGill University

\section{Corresponding authors:}

Dr Holly Bridge

holly.bridge@ndcn.ox.ac.uk

FMRIB Centre

John Radcliffe Hospital

Oxford

OX3 9DU

UK

Title: 59 characters

Running Head: 26 characters

Abstract: 97 words

Body of manuscript:1600

Number of color figures: 2

Number of tables: 1
Dr Zameel Cader

zameel.cader@ndcn.ox.ac.uk

Level 6, West Wing

John Radcliffe Hospital

Oxford

OX3 9DU

UK 


\section{Abstract:}

Background: Visual aura is present in about one-third of migraine patients and triggering by bright or flickering lights is frequently reported.

Method: Using migraine with visual aura patients, we investigated the neurochemical profile of the visual cortex using magnetic resonance spectroscopy. Specifically, Glutamate/Creatine and GABA/Creatine ratios were quantified in the occipital cortex of female migraine patients.

Results: GABA levels in the occipital cortex of migraine patients were lower than control subjects. Glutamate levels in migraine patients, but not controls, correlated with the blood-oxygenation-level-dependent (BOLD) signal in the primary visual cortex during visual stimulation.

Conclusion: Migraine with visual aura appears to disrupt the excitation-inhibition coupling in the occipital cortex.

Key words: Migraine; magnetic resonance spectroscopy; GABA; glutamate; visual cortex 


\section{Introduction}

Migraine is intimately linked to the visual system, associated with visual auras, photophobia and visual triggering of attacks. Halogen bulbs, flicker or bright sunlight are well-described triggers for migraine attacks in addition to more standard triggers such as tiredness or stress (1). A recent study has suggested that visual stimuli may not be a reliable migraine provocant, but this may be very dependent on the nature of the stimulus used and the state of the patient. The vulnerability of the visual system in patients with migraine may reflect deficits in excitatory-inhibitory balance, with several studies of rare genetic forms of migraine affecting both glutamatergic and GABA-ergic synapses (2). Disturbances in excitatory-inhibitory balance may also predispose the cortex to cortical spreading depression (CSD), a phenomenon thought to underlie migraine aura (3).

${ }^{1} \mathrm{H}-\mathrm{MRS}$ can be used to quantify a number of neurochemicals within a specific region of the brain, and current MRS protocols permit the detection of GABA and glutamate peaks in addition to metabolites that are found in higher concentrations (4). No previous studies have succeeded in quantifying GABA levels in migraine patients, but studies have suggested that there may be abnormalities in glutamatergic cycling. Prescot et al. (2009) (5), using linear discriminant analysis, suggested a different pattern of NAA and glutamine concentrations in the migraine subjects compared to controls. In a second study, Siniatchkin et al. (2012) (6) showed that young MA patients had significantly higher glutamate + glutamine $(\mathrm{Glx}) /$ Creatine $(\mathrm{Cr})$ ratios than healthy subjects. Furthermore, while application of excitatory (anodal) transcranial direct current stimulation (tDCS) to the visual cortex in healthy subjects caused an increase in $\mathrm{Glx} / \mathrm{Cr}$ ratio and inhibitory (cathodal) stimulation caused a decrease, both types of tDCS caused a decrease in $\mathrm{Glx} / \mathrm{Cr}$ ratio in migraine patients, Finally, a recent paper by Aleja (7) showed an increase in the ratio of occipital Glutamate: Glutamine. Taken together these findings suggest disruption of normal glutamate turnover and function without changes in glutamate levels.

The comprehensive review of Reyngoudt et al, (8) highlights the lack of studies that recruited homogeneous patient types with control groups matched for age and gender, meaning that MRS results to date are difficult to interpret. Here, we scanned female patients with migraine with visual aura (vMA) and age and gender-matched controls subjects using a MRS sequence that allowed accurate quantification of GABA, glutamate and glutamine. 


\section{Methods}

Twenty-six female participants were recruited to the study conducted under ethical approval from the Staffordshire NHS Research Ethics Committee (11/WM/0088), and all subjects provided informed written consent. Thirteen subjects (mean age $33 \pm 8$ years), with migraine with visual aura (citing visual stimuli as potent migraine triggers), were recruited via clinics in Oxford, personal contacts and via an advertisement placed on the 'Migraine Trust' website. All potential participants were clinically assessed by a migraine clinician (ZC) to ensure that they met the eligibility criteria for the study: revised International Headache Society criteria for migraine with aura; predominant aura was visual; and visual stimulation a strong selfreported trigger for migraine episodes.

Thirteen female control subjects, with no history of migraine or other primary headache disorder, were recruited via email advertisement and through personal contacts (mean age $30 \pm 6$ years). The migraine characteristics of the patients are shown in SI Table 1.

A single imaging session was performed at the Oxford Centre for Functional Magnetic Resonance Imaging of the Brain (FMRIB) using a Siemens 3T Verio scanner with a 32channel head coil. The scan room was dark and subjects wore light diffusing goggles. The first scan was a whole-head structural scan (magnetization prepared rapid gradient echo, repetition time $=11.2 \mathrm{~ms}$, echo time $=4.68 \mathrm{~ms}$, resolution $1 \times 1 \times 1 \mathrm{~mm}^{3}$ ). Magnetic resonance spectroscopy (MRS) used a single voxel manually located to cover V1 bilaterally. The SPECIAL (SPin ECho full Intensity Acquired Localized spectroscopy) sequence (9) was employed using the following standard parameters: VOI $=20 \times 20 \times 20 \mathrm{~mm}^{3}, \mathrm{TR} / \mathrm{TE}=4000$ $\mathrm{ms} / 8.5 \mathrm{~ms}$, number of averages $=128$. The short echo-time single voxel acquisition enables simultaneous detection of many metabolites, including glutamate and GABA $(9,10)$.

Standard pre-processing to correct for motion and frequency drift was applied using in-house scripts as described previously (11), and all spectra used for analysis had a linewidth $<6 \mathrm{~Hz}$ (average $4.8 \mathrm{~Hz}$; no group difference). Metabolite quantification was performed using LCModel (Provencher, 1993), and all metabolites were fitted with Cramer-Rao Lower Bounds of $<20 \%$. As is customary, all neurotransmitter levels are presented as a ratio to total Creatine $(\mathrm{Cr}+\mathrm{PCr})$, a simultaneously-acquired reference metabolite. For each participant the T1-weighted structural image was segmented using FAST (FMRIB's automated segmentation tool) (12), and the relative grey matter, white matter and cerebrospinal fluid contributions to the MRS voxel was calculated and used to correct MRS values for grey matter volume (13). 
BOLD activity in primary visual cortex was quantified in two fMRI experiments. In experiment 1 , subjects were presented with diffuse light for $120 \mathrm{~s}$ followed by $60 \mathrm{~s}$ in the dark. This was repeated 3 times, and 'light onset' was defined as the first $30 \mathrm{~s}$ of diffuse light in each repeat. In experiment 2 participants were presented with $15 \mathrm{sec}$ blocks of either 'high' $(20 \%$ and $50 \%)$ or 'low' ( $2 \%$ and $5 \%)$ contrast flickering checkerboard stimuli or a uniform gray background with a fixation cross. Subjects were explicitly asked to keep their eyes open for the duration of both fMRI experiments. Two control subjects were excluded from the checkerboard experiment, as the mirror was not correctly positioned.

\section{$\underline{\text { Results }}$}

Spectra of sufficient quality to determine GABA and glutamate levels were obtained from 11 subjects in each group. In line with previous work, we did not find any difference in glutamate levels between patients and controls (Figure $1 \mathrm{C}, \mathrm{t}(21)=0.85 ; \mathrm{p}=0.41$ ). GABA levels, however, were $\sim 10 \%$ lower in migraine patients than in controls (Figure 1D, 1-way, $\mathrm{t}(21)=1.8 ; \mathrm{p}=0.042)$.

\section{Occipital glutamate correlates with BOLD signal in migraine patients, but not controls}

To determine whether there was any relationship to visual activation in the occipital cortex, BOLD responses to two different types of light stimulation were correlated to levels of glutamate and GABA. Figure 2A shows the relationship of glutamate level with 'light onset', which was not significant in migraine patients $(r=0.47 ; p=0.13)$ or controls $(r=-0.04 ; p=$ 0.91). In contrast, $\mathrm{B}$ and $\mathrm{C}$ show the correlation with low and high contrast flickering checkerboards respectively. There was a highly significant correlation in migraine patients (filled black circles) in both contrasts (low: $r=0.74 ; p<0.01$; high: $r=0.88 ; p<0.0005$ ), although it should be noted that, unsurprisingly, the low and high contrast responses are correlated. The equivalent correlations in control subjects are not significant (low contrast: $r$ $=0.1 ; \mathrm{p}=0.8 ;$ high contrast: $\mathrm{r}=0.06 ; \mathrm{p}=0.87$ ). The correlation in the high contrast condition was significantly greater in migraine patients than controls (Fisher r-to-z transform; $p=$ 0.015 ), and close to significance in the low condition (Fisher $r$-to-z transform; $p=0.056$ ).

There was no correlation between BOLD signal and GABA concentration in either migraine or control subjects in any visual condition. This remained the case even when glutamate concentration was partially correlated out of the calculation.

\section{Discussion}


This homogeneous population of vMA patients shows decreased GABA levels in the occipital cortex relative to matched control subjects. While resting GABA levels have been linked to orientation discrimination, gamma oscillation frequency and BOLD activation in the visual cortex of healthy controls (14), this relationship did not reach significance in the control subjects in this study. This could be due either to small subject numbers or to a difference in sensitivity in GABA detection between the SPECIAL sequence and the MEGA-PRESS used previously.

MRS-GABA has been suggested to be a measure of extra-synaptic tonic GABAergic activity (4), and therefore the reduced GABA levels observed here in migraineurs could most easily be interpreted as a reduction in local inhibition. This finding is consistent with several studies suggesting occipital hyper-excitability in migraine (15). However we note that in our subjects, BOLD responses to light onset and checkerboard stimulation were not increased relative to controls, suggesting any change in excitability may be subtle, complex and perhaps not homogenous across the occipital cortex.

Consistent with at least one previous study (7), we found no difference in glutamate levels between migraineurs and controls. However, we demonstrated a significant positive relationship between occipital glutamate levels and the BOLD response to visual stimulation in migraineurs, a relationship that was not seen in the control subjects. This finding provides further support for the hypothesis of disrupted excitatory-inhibitory coupling in migraine (2). A close relationship between BOLD signal and glutamate levels, has not previously been demonstrated in the visual cortex of migraine patients or of controls, but has been shown in the anterior cingulate cortex in major depression (16).

Total glutamate, as assessed by MRS, reflects glutamate's role as a metabolite as well as a neurotransmitter. Significant changes may occur in glutamatergic transmission in migraineurs, which drive the correlation between the BOLD signal and glutamate in this group, but is not visible using this technique. However, this interpretation remains speculative. Although a causal relationship remains to be established, our data suggest that, in addition to GABA, glutamate may be a key driver of occipital activation in migraine with aura subjects. Due to time constraints, we did not acquire data from an additional voxel in another cortical region, so it is not possible to determine whether the observed changes are confined to the occipital lobe or are more widespread. Nevertheless, disturbance of glutamate metabolism and neurotransmission is also supported by genetic studies (17). The extent to which our findings are aura specific or more generally migraine-related, will require further studies comparing migraine with aura and migraine without aura subjects. This could also 
address the important question of whether altered neurochemical coupling is a feature of light sensitivity or visual aura.

In conclusion, a homogenous group of vMA patients show reduced GABA and enhanced glutamate-cortical activation coupling in the occipital cortex, which may predispose subjects to visual auras and migraine triggering by visual stimuli. It is notable that Topiramate, a first line migraine prophylactic, is a glutamate receptor antagonist (18), enhances GABA activity (19) and increases brain GABA (20), although this is not the case for all migraine prophylactics. Future studies should investigate the causal relationship between these neurotransmitters and cortical activity in migraine, through pharmacological manipulation of GABA and glutamate signalling. Nevertheless the observed change in excitatory-inhibitory coupling is an important signature of the migraine brain and highlights therapeutic opportunities.

\section{Article Highlights}

- Female patients with migraine with visual aura and visual sensitivity show decreased GABA in the occipital cortex

- Occipital Glutamate/Creatine ratio correlates with BOLD activation levels in primary visual cortex

\section{References}

[1] Harle DE, Shepherd AJ, Evans BJ. Visual stimuli are common triggers of migraine and are associated with pattern glare. Headache. 2006;46(2006):143140.

[2] Vecchia D, Pietrobon D. Migraine: a disorder of brain excitatory-inhibitory balance? Trends in neurosciences. 2012;35(2012):507-20.

[3] Hadjikhani N, Sanchez Del Rio M, Wu O, Schwartz D, Bakker D, Fischl B, et al. Mechanisms of migraine aura revealed by functional MRI in human visual cortex. Proceedings of the National Academy of Sciences of the United States of America. 2001;98(2001):4687-92. 
[4] Stagg CJ, Bachtiar V, Johansen-Berg H. What are we measuring with GABA magnetic resonance spectroscopy? Communicative \& integrative biology. 2011;4(2011):573-5.

[5] Prescot A, Becerra L, Pendse G, Tully S, Jensen E, Hargreaves R, et al. Excitatory neurotransmitters in brain regions in interictal migraine patients. Molecular pain. 2009;5(2009):34.

[6] Siniatchkin M, Sendacki M, Moeller F, Wolff S, Jansen O, Siebner H, Stephani U. Abnormal changes of synaptic excitability in migraine with aura. Cerebral cortex. 2012;22(2012):2207-16.

[7] Gonzalez de la Aleja J, Ramos A, Mato-Abad V, Martinez-Salio A, Hernandez-Tamames JA, Molina JA, et al. Higher glutamate to glutamine ratios in occipital regions in women with migraine during the interictal state. Headache. 2013;53(2013):365-75.

[8] Reyngoudt H, Achten E, Paemeleire K. Magnetic resonance spectroscopy in migraine: what have we learned so far? Cephalalgia : an international journal of headache. 2012;32(2012):845-59.

[9] Mekle R, Mlynarik V, Gambarota G, Hergt M, Krueger G, Gruetter R. MR spectroscopy of the human brain with enhanced signal intensity at ultrashort echo times on a clinical platform at 3T and 7T. Magnetic resonance in medicine: official journal of the Society of Magnetic Resonance in Medicine / Society of Magnetic Resonance in Medicine. 2009;61(2009):1279-85.

[10] Near J, Andersson J, Maron E, Mekle R, Gruetter R, Cowen P, Jezzard P. Unedited in vivo detection and quantification of gamma-aminobutyric acid in the occipital cortex using short-TE MRS at 3 T. NMR in biomedicine. 2013;26(2013):1353-62.

[11] Near J, Edden R, Evans CJ, Paquin R, Harris A, Jezzard P. Frequency and phase drift correction of magnetic resonance spectroscopy data by spectral registration in the time domain. Magnetic resonance in medicine : official journal of the Society of Magnetic Resonance in Medicine / Society of Magnetic Resonance in Medicine. 2014(2014).

[12] Zhang Y, Brady M, Smith S. Segmentation of brain MR images through a hidden Markov random field model and the expectation-maximization algorithm. IEEE transactions on medical imaging. 2001;20(2001):45-57. 
[13] Stagg CJ, Wylezinska M, Matthews PM, Johansen-Berg H, Jezzard P, Rothwell JC, Bestmann S. Neurochemical effects of theta burst stimulation as assessed by magnetic resonance spectroscopy. Journal of neurophysiology. 2009;101(2009):2872-7.

[14] Edden RA, Muthukumaraswamy SD, Freeman TC, Singh KD. Orientation discrimination performance is predicted by GABA concentration and gamma oscillation frequency in human primary visual cortex. The Journal of neuroscience : the official journal of the Society for Neuroscience. 2009;29(2009):15721-6.

[15] Hougaard A, Amin FM, Hoffmann MB, Rostrup E, Larsson HB, Asghar MS, et al. Interhemispheric differences of fMRI responses to visual stimuli in patients with side-fixed migraine aura. Human brain mapping. 2014;35(2014):2714-23. [16] Walter M, Henning A, Grimm S, Schulte RF, Beck J, Dydak U, et al. The relationship between aberrant neuronal activation in the pregenual anterior cingulate, altered glutamatergic metabolism, and anhedonia in major depression. Archives of general psychiatry. 2009;66(2009):478-86.

[17] Anttila V, Winsvold BS, Gormley P, Kurth T, Bettella F, McMahon G, et al. Genome-wide meta-analysis identifies new susceptibility loci for migraine. Nature genetics. 2013;45(2013):912-7.

[18] Braga MF, Aroniadou-Anderjaska V, Li H, Rogawski MA. Topiramate reduces excitability in the basolateral amygdala by selectively inhibiting GluK1 (GluR5) kainate receptors on interneurons and positively modulating GABAA receptors on principal neurons. The Journal of pharmacology and experimental therapeutics. 2009;330(2009):558-66.

[19] Angehagen M, Ben-Menachem E, Ronnback L, Hansson E. Novel mechanisms of action of three antiepileptic drugs, vigabatrin, tiagabine, and topiramate. Neurochemical research. 2003;28(2003):333-40.

[20] Kuzniecky R, Ho S, Pan J, Martin R, Gilliam F, Faught E, Hetherington H. Modulation of cerebral GABA by topiramate, lamotrigine, and gabapentin in healthy adults. Neurology. 2002;58(2002):368-72. 


\section{Funding}

This work was funded by the Medical Research Council (UK), the Royal Society and The John Fell Fund. 
Figure 1. MRS data were acquired from a voxel centered on the calcarine sulcus as shown in A. An example spectrum with glutamate and one of the GABA peaks indicated is shown in B. Concentrations of glutamate and GABA normalized to creatine are shown in C and D respectively.

Figure 2. A shows the correlation between the response to the onset of diffuse, dim light in V1 and normalized occipital glutamate concentration. Filled black circles are migraine patients and open circles are control participants. The black line shows the least squares fit to the migraine patients and the gray line shows the fit to the control subjects. B and C show the correlations of glutamate with the $\mathrm{V} 1$ responses to low contrast and high contrast flickering checkerboards.

A Mean voxel location

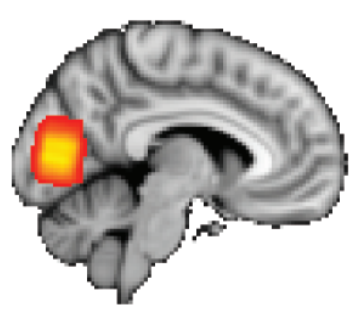

C

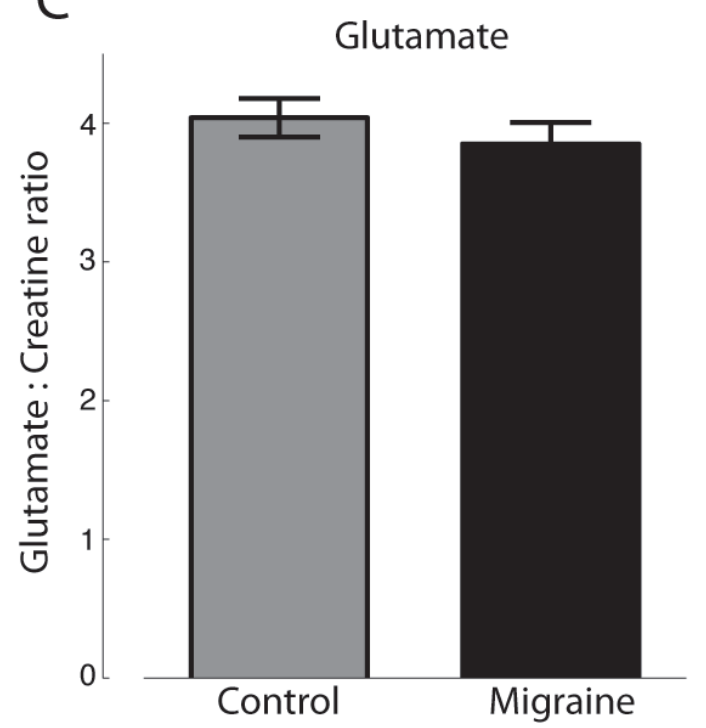

B

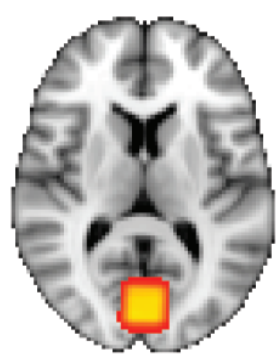

D

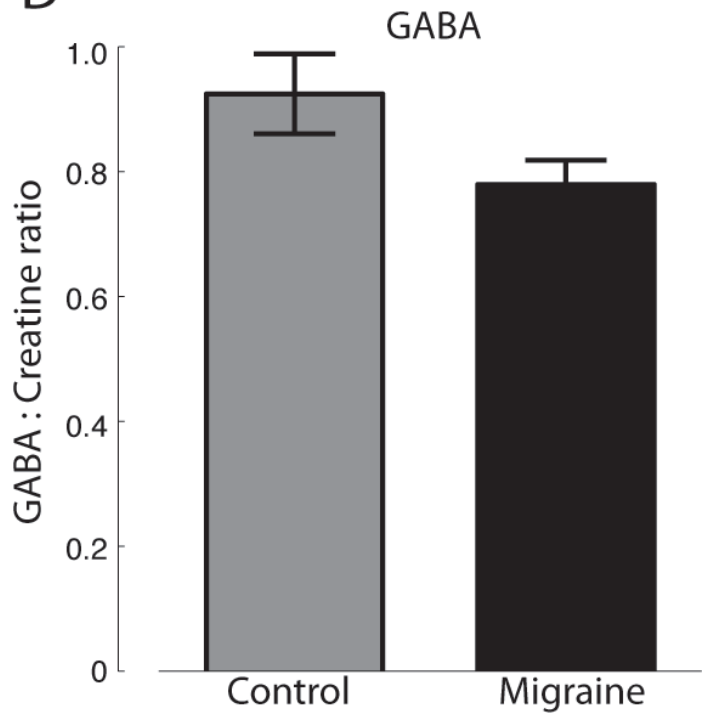


A

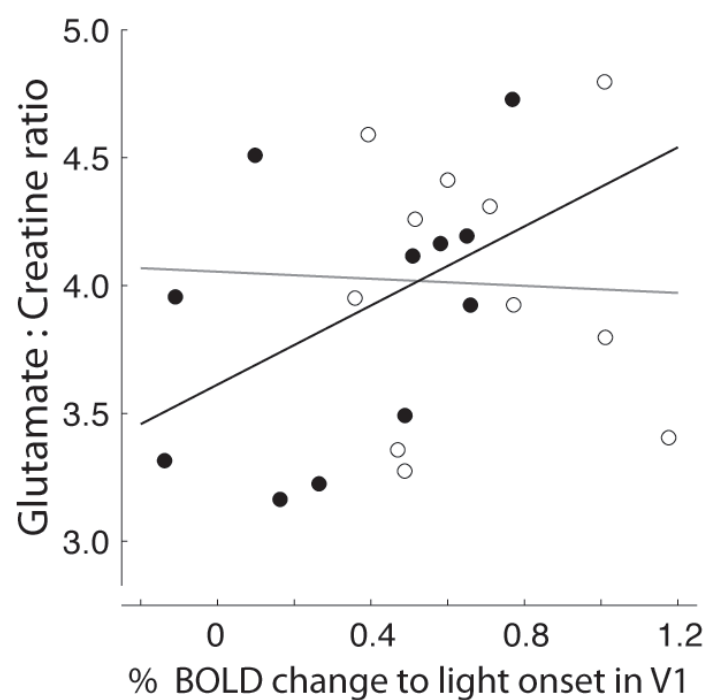

B

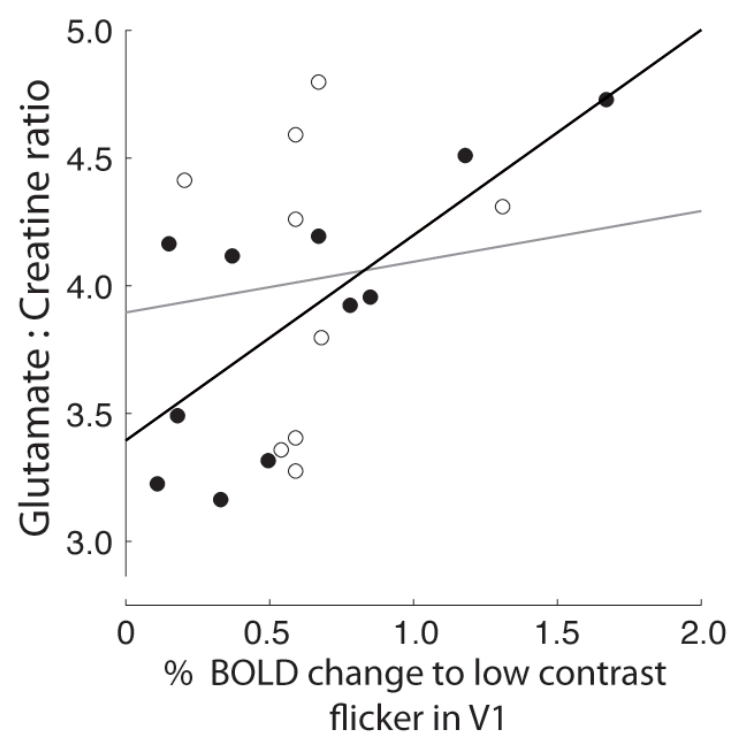

C

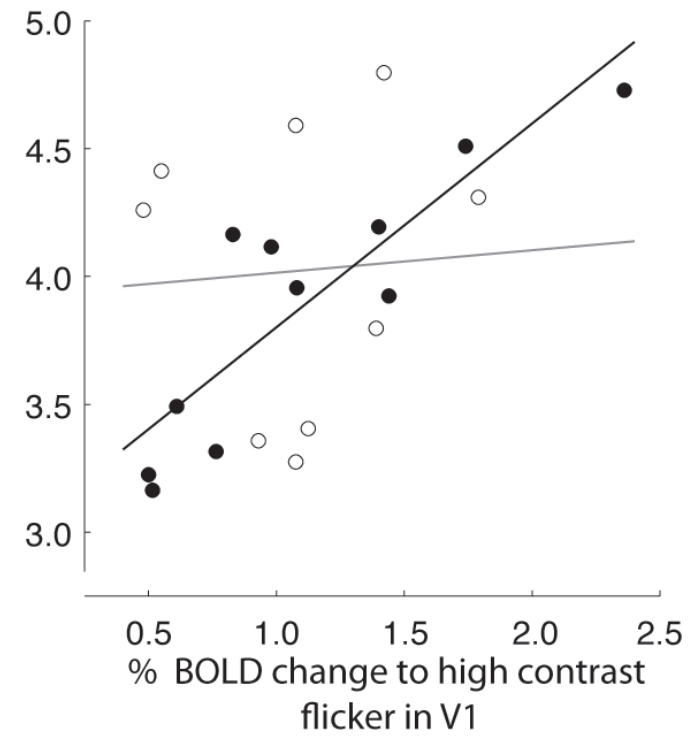

